\title{
Distribution des types de virus du papillome humain, antécédents de dépistage du cancer du col de l'utérus et facteurs de risque d'infection au Manitoba
}

\author{
A. A. Demers, Ph. D. (1,2,3); B. Shearer, Ph. D. (4); A. Severini, M.D. (5); R. Lotocki, M.D., FRCSC (6,7); \\ E. V. Kliewer, Ph. D. (2,3,8); S. Stopera, M. Sc. (9); T. Wong, M.D., FRCPC (1,10); G. Jayaraman, Ph. D. (1,11)
}

Cet article a fait l'objet d'une évaluation par les pairs.

\section{Résumé}

Objectifs : L’objectif de cette étude est l'analyse de la prévalence des infections dues au virus du papillome humain (VPH) dans un échantillon opportuniste de femmes résidant au Manitoba (Canada). Nous avons examiné les facteurs de risque associés aux infections par le VPH et avons établi des liens entre les résultats du typage du VPH et les antécédents de dépistage du cancer du col utérin des participantes.

Méthode : La population étudiée comprenait 592 femmes ayant fréquenté une clinique de dépistage utilisant le test de Papanicolaou (Pap). Après avoir signé un formulaire de consentement, les participantes répondaient à un questionnaire auto-administré sur les facteurs de risque et ont subi un test de Pap classique. Les cellules résiduelles des tests de Pap ont été recueillies et envoyées pour un typage du VPH.

Résultats : Lâge moyen de la population était de 43 ans. En tout, 115 participantes (19,4 \%) étaient infectées par le VPH, 89 d'entre elles ayant obtenu un résultat normal au test de Pap. Parmi celles qui étaient positives pour le VPH, 61 (10,3 \%) étaient infectées par un VPH à risque élevé (Groupe 1). Le VPH-16 était le type le plus répandu (15/115 : 13,0 \% des infections). Les facteurs de risque d'infection par le VPH les plus fréquents étaient le jeune âge, l'origine autochtone, un nombre plus élevé de partenaires sexuels au cours de la vie et un nombre plus élevé de partenaires sexuels au cours de l’année précédente.

Conclusions : La prévalence des types de VPH au Manitoba correspond aux distributions signalées dans d'autres provinces ou territoires. Ces données fournissent des renseignements de base sur la prévalence du VPH de chaque type dans une population non vaccinée et peuvent aider à évaluer l'efficacité du programme de vaccination contre le VPH. En outre, ces données permettent d'effectuer une validation de principe du couplage d'un registre de tests de Pap basé sur une population avec les résultats de tests de laboratoire et une enquête sur les comportements à risque en vue d'évaluer les effets précoces et tardifs de l'infection par le VPH. Cette méthodologie pourrait être appliquée à d'autres provinces ou territoires au Canada disposant de ressources similaires.

Mots clés : infections par le virus du papillome humain, prévalence, facteurs de risque, dysplasie du col de l'utérus, détection précoce du cancer

\section{Introduction}

Les programmes publics de vaccination contre le virus du papillome humain (VPH) mis en œuvre dans tout le Canada entre 2007 et 2009 offrent la possibilité de prévenir une grande proportion de condylomes anogénitaux, de lésions cervicales de haut grade et de cancers envahissants liés au $\mathrm{VPH}^{1-6}$. Ils peuvent également influencer les pratiques actuelles de dépistage du cancer du col utérin du fait des changements dans la prévalence des anomalies cervicales qu'ils peuvent entraîner ${ }^{1,7}$. Le degré d'impact de ces

Rattachement des auteurs :

1. Centre de la lutte contre les maladies transmissibles et les infections, Agence de la santé publique du Canada, Ottawa (Ontario), Canada

2. Département d'épidémiologie et Registre du cancer, ActionCancer Manitoba, Winnipeg (Manitoba), Canada

3. Département des sciences de la santé communautaire, Université du Manitoba, Winnipeg (Manitoba), Canada

4. Centre international des maladies infectieuses, Winnipeg (Manitoba), Canada

5. Laboratoire national de microbiologie, Agence de la santé publique du Canada, Winnipeg (Manitoba), Canada

6. Oncologie gynécologique, ActionCancer Manitoba, Winnipeg (Manitoba), Canada

7. Programme de dépistage du cancer de l'utérus du Manitoba (Manitoba), Canada

8. Cancer Control Research, British Columbia Cancer Agency, Vancouver (Colombie-Britannique), Canada

9. Direction de la lutte contre les maladies transmissibles, Santé publique et soins de santé primaires, ministère de la Santé du Manitoba, Winnipeg (Manitoba), Canada

10. Département des maladies infectieuses, Université d'Ottawa, Ottawa (Ontario), Canada

11. Département d'épidémiologie et de médecine communautaire, Université d'Ottawa, Ottawa (Ontario), Canada

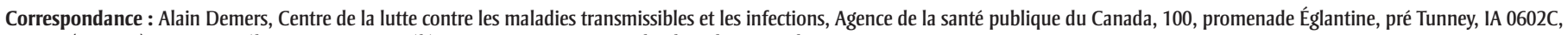
Ottawa (Ontario) K1A 0K9; tél. : 613-948-8247; téléc. : 613-941-9813; courriel : alain.demers@phac-aspc.gc.ca 
programmes dépendra cependant de la distribution des types de VPH, des taux d'infection par type chez les femmes et de la participation aux programmes de vaccination.

L’objectif de cette étude était de déterminer la prévalence de base pour chaque type de virus et les facteurs de risque d'infection par le VPH dans un échantillon opportuniste de femmes fréquentant des cliniques sans rendez-vous au Manitoba (Canada) qui offraient le test de Papanicolaou (Pap) durant une semaine annuelle de sensibilisation au cancer du col de l'utérus. Les données de l'enquête et les résultats du typage du VPH ont été couplés à la base de données du Programme de dépistage du cancer du col de l'utérus du Manitoba (PDCCUM). Le Manitoba est bien placé pour parrainer et effectuer ce type de projet de surveillance parce qu'il dispose de bases de données en population sur le cancer, sur le dépistage du cancer du col de l'utérus, sur les interventions médicales pratiquées par les médecins et sur la vaccination $^{8}$. Ces ressources fournissent des outils solides pour évaluer l'impact du programme de vaccination contre le VPH, l'utilisation du dépistage du cancer du col de l'utérus chez les femmes vaccinées et non vaccinées ainsi que la distribution et les conséquences résultant de la maladie.

\section{Méthode}

\section{Cadre de l'étude}

Depuis 2003, le PDCCUM organise une semaine annuelle de test de Pap en octobre. Au cours de cette semaine, les femmes sont invitées à se présenter aux cliniques de dépistage par test de Pap sans rendez-vous. L'objectif est d'atteindre celles qui n'ont jamais subi ou n'ont pas subi régulièrement de dépistage du cancer du col de l'utérus. En 2008, 123 cliniques ont participé à la Semaine manitobaine du test de Pap. Parmi elles, 52 ont accepté de participer à cette étude. En plus d'effectuer les tests de Pap classiques, ces cliniques ont recueilli les cellules résiduelles des tests de Pap, les ont placées dans un milieu liquide pour un examen cytologique et ont expédié les échantillons au Laboratoire provincial
Cadham à Winnipeg (Manitoba) pour un typage du VPH. Les cliniques participantes ont également supervisé l'administration d'un formulaire de consentement et d'un questionnaire auto-administré sur les facteurs de risque d'infection au VPH.

\section{Population}

La population étudiée était composée d'un échantillon opportuniste de femmes âgées de 18 ans et plus provenant de différents milieux ethniques. Les femmes enceintes ont été exclues. Les femmes intéressées à participer à l'étude ont discuté des objectifs avec un employé de la clinique et, après avoir accepté de participer, ont signé un formulaire de consentement et rempli un questionnaire sur les facteurs de risque. Les femmes qui ont décidé de ne pas remplir le questionnaire demeuraient admissibles au test de dépistage du VPH, et leurs résultats ont été inclus dans l'analyse.

De la publicité a été faite sur l'étude au moyen d'affiches dans les cliniques, et les employés en ont parlé aux participantes potentielles. En tout, 1182 femmes ont subi un dépistage du cancer du col de l'utérus dans les 52 cliniques participantes, et 642 (54\%) ont consenti à participer à l'étude.

\section{Suivi des participantes}

Des professionnels de la santé ont reçu les résultats des tests de Pap et du typage du VPH. Les participantes ayant reçu un diagnostic d'anomalies cervicales ont été suivies conformément aux lignes directrices pour la prise en charge des cas dépistés de cancer du col de l'utérus du PDCCUM qui étaient en vigueur au moment de l'étude. Les femmes qui étaient positives pour le VPH à risque élevé et négatives à l'examen cytologique ont été rappelées par les cliniques pour subir un examen plus approfondi, conformément aux lignes directrices du PDCCUM.

\section{Enquête sur les facteurs de risque}

L'enquête comportait des questions sur les caractéristiques socio-démographiques et les facteurs de risque pertinents de néoplasie cervicale, notamment l'usage du tabac, celui de contraceptifs oraux, l'activité sexuelle récente, le diagnostic antérieur d'infections transmises sexuellement et le statut à l'égard de la vaccination contre le VPH. Avant d'être utilisé, le questionnaire a été testé pour des lecteurs de niveau de quatrième année.

\section{Traitement des échantillons cervicaux et détection et typage du VPH}

La méthode Luminex est une technique mise au point par le Laboratoire national de microbiologie et qui permet de détecter 45 types de VPH, dont 23 des 25 types à risque élevé (définis par le Centre international de recherche sur le cancer) appartenant aux Groupes 1, 2a et $2 \mathrm{~b}$ : types $16,18,26,30,31,33,35,39,45,51$, $52,53,56,58,59,66,67,68,69,70,73$, 82 et $85 \mathrm{du} \mathrm{VPH}^{9}$. On retrouve également 22 types considérés comme à faible risque ou à risque inconnu : types $6,11,13,32$, $40,42,43,44,54,61,62,71,72,74,81$, 83, 84, 86, 87, 89, 90 et 91 du VPH. Pour résumer, les échantillons placés dans un milieu de transport viral ont été centrifugés et leur ADN a été extrait du culot résultant à l'aide d'une trousse d'extraction de l'ADN MagnaZorb ${ }^{10,11}$. L'ADN a été amplifié par polymérase (PCR) nichée, la série générale d'amorces PGMY étant utilisée pour le premier cycle $^{12}$ et la série d'amorces GP5 + /GP6 + pour le second $\operatorname{cycle}^{13}$. Cette méthode permet d'amplifier un fragment de la région L1 du génome du VPH (d'environ 150 paires de bases en longueur). La qualité de l'échantillon d'ADN pour la PCR a été vérifiée par co-amplification du gène de la bêta-globine humaine. Les produits de la PCR ont été détectés visuellement par électrophorèse sur gel ${ }^{13-16}$.

L'ADN du VPH a été détecté et typé par hybridation sur des microsphères couplées à des sondes spécifiques pour les 45 types de VPH selon la technologie xMAP Luminex* La spécificité et la sensibilité de cette méthode ont été mesurées pour les 45 types de VPH au moyen d'ADN de VPH clonés. Une comparaison entre la trousse LinearArray (Roche) ${ }^{17}$ et d'autres trousses de génotypage 
du VPH a révélé que la méthode Luminex était équivalente aux autres méthodes commerciales de génotypage ${ }^{18}$.

\section{Analyse des données}

Nous avons couplé les résultats du typage du VPH et les résultats d'enquête avec la base de données du PDCCUM en utilisant un identificateur unique afin d'obtenir les résultats des tests de Pap effectués durant la Semaine du test de Pap 2008 ainsi que les antécédents de dépistage du cancer du col de l'utérus des participantes consentantes. Des analyses de régression logistique unidimensionnelle et multidimensionnelle ont servi à calculer les rapports de cotes (RC) et les intervalles de confiance (IC) à $95 \%$ utilisés comme estimations du risque relatif de détection du VPH associé aux diverses variables prédictives. Comme la prévalence du VPH est plus élevée chez les femmes de moins de 30 ans, les résultats ont été compilés en différenciant ceux pour les femmes de moins de 30 ans et ceux pour les femmes de 30 ans et plus. Les types de VPH ont été regroupés selon la méthode de Bouvard et collab ${ }^{9}$. et de Villiers et collab. ${ }^{19}$.

Le protocole a été approuvé par les comités d'éthique pour la recherche de Santé Canada/Agence de la santé publique du Canada et de l'Université du Manitoba.

\section{Résultats}

Les échantillons de tissus prélevés chez les 642 femmes qui ont consenti à participer à l'étude ont été soumis à un test de dépistage de l'infection par le VPH. Parmi les participantes, 33 n'ont pas rempli le formulaire de consentement et ont été exclues des analyses. Dix-sept autres ont été exclues parce que leurs échantillons étaient inadéquats. La population de l'étude comptait donc 592 participantes, dont 527 ayant répondu au questionnaire. L'âge moyen de la population étudiée était de 43 ans (âge médian : 44). L'âge moyen des femmes infectées était de 35 ans (âge médian : 31 ans), et l'âge moyen des femmes non infectées était de 45 ans (âge médian : 46 ans). La majorité des participantes provenaient de régions rurales $(66,3 \%)$ et le reste de Winnipeg et de Brandon.

\section{Résultats de l'enquête}

Les variables associées à l'infection par le VPH ont fait l'objet d'une analyse unidimensionnelle et sont présentées dans le tableau 1. Les résultats ont été ventilés entre les femmes de moins de 30 ans (appelées "plus jeunes ») et celles de 30 ans et plus (appelées "plus âgées ») pour tenir compte de la plus forte prévalence des infections à VPH chez les plus jeunes. Chez les plus âgées, l'infection par le VPH était associée à une origine autochtone et une situation financière décrite par les participantes comme étant difficile. Comparativement aux non-fumeuses, les participantes qui fumaient couraient un plus grand risque d'être positives pour le VPH, quel que soit l'âge. L'absence d'antécédents d'infections transmises sexuellement (ITS) protégeait contre l'infection par le VPH dans les deux groupes d'âge. Les femmes qui comptaient un plus grand nombre de partenaires sexuels au cours de leur vie ou un plus grand nombre de partenaires sexuels au cours de l'année écoulée étaient plus nombreuses à être positives pour le VPH. Les femmes plus jeunes qui n'entretenaient pas de relation stable étaient plus nombreuses à être positives pour le VPH que celles qui avaient une relation stable ou étaient plus âgées.

Dans l'analyse de régression logistique multidimensionnelle, le fait d'être plus jeune $(\mathrm{RC}=0,97$; IC à $95 \%: 0,95$ à 0,99; l'âge étant traité comme une variable continue), d'avoir une origine autochtone $(\mathrm{RC}=4,83$; IC à $95 \%: 2,70$ à 8,65 ; comparativement à l'origine non autochtone) et d'avoir eu deux partenaires sexuels ou plus au cours de l'année écoulée $(\mathrm{RC}=2,04$; IC à $95 \%: 1,20$ à 3,47 ; comparativement à un ou aucun partenaire sexuel) étaient des prédicteurs significatifs de résultats positifs pour le VPH. Les variables qui n'étaient pas des prédicteurs significatifs de l'infection par le VPH dans le modèle multidimensionnel étaient les suivantes : l'usage actuel de tabac (oui/non), des antécédents de tests de Pap (oui/non), des antécédents d'anomalies cervicales (oui/non) et le fait d'avoir eu au moins deux examens de dépistage consécutifs au cours d'une année (oui/non).
Antécédents de test de Pap selon les déclarations et d'après le registre

Les femmes plus âgées qui n'avaient subi aucun test de Pap entre 2001 (l'année où la base de données du PDCCUM a débuté) et octobre 2008 couraient un plus grand risque d'être positives pour le VPH (tableau 1; données du PDCCUM). Une tendance similaire a été observée pour les antécédents déclarés de test de Pap, bien que le nombre de répondantes n'ayant jamais subi de test de Pap ait été faible. Les femmes plus jeunes qui ont déclaré n'avoir jamais subi de test de Pap couraient un risque plus faible d'être infectées par le $\mathrm{VPH}$, mais cette tendance n'a pas été observée lorsque les analyses ont porté sur les données du PDCCUM.

\section{Infections par le VPH et résultats cytologiques}

En tout, 115 participantes (19\%) se sont révélées positives au test d'infection par VPH (tableau 2). Dans l'ensemble, $33 \%$ (38/115) de ces infections ont été détectées chez des participantes âgées de moins de 25 ans. Les femmes de ce groupe d'âge étaient également plus nombreuses à être infectées par les types de VPH du Groupe 1 (44 \%; 27/61) que par les types du Groupe 2 (21 \%; 4/19). Les types 6 et 11 du VPH n'ont pas été détectés chez les femmes de 30 ans et plus, alors que les types 16 et 18 (mais surtout 16) ont été détectés dans un plus large éventail d'âges. Un quart des femmes infectées (26\%; 30/115) présentaient de multiples infections par le VPH, c'est-à-dire qu'elles étaient infectées par plus d'un type de VPH.

Parmi les femmes de l'étude ayant obtenu un résultat normal au test de Pap, 17 \% (89/517) étaient positives pour une infection par le VPH, et 9 \% (46/517) étaient infectées par un VPH du Groupe 1 (tableau 3). Dans l'ensemble, 7 \% (41/592) de toutes les participantes ont obtenu un résultat anormal au test de Pap. Une infection par le VPH (tous types confondus) a été décelée chez $11 \%$ des participantes ayant obtenu des résultats insatisfaisants (2/18), $32 \%$ de celles qui présentaient une atypie des cellules malpighiennes de signification indéterminée (ASC-US; 6/19), 63 \% des cas de lésion malpighienne intra-épithéliale 
TABLEAU 1

Résultats d'enquête selon l'âge et le statut à l'égard de l'infection par le VPH

\begin{tabular}{|c|c|c|c|c|c|c|c|c|c|}
\hline \multirow[t]{3}{*}{ Variables $^{\mathrm{a}}$} & \multirow[t]{3}{*}{ Categories } & \multicolumn{3}{|c|}{ Âge $<30$ ans } & \multicolumn{5}{|c|}{ Âge $\geq 30$ ans } \\
\hline & & \multirow{2}{*}{$\begin{array}{c}\text { VPH }-(n=75) \\
\text { n } \%\end{array}$} & $\mathrm{VPH}+(\mathrm{n}=56)$ & \multirow{2}{*}{ RC (IC à 95 \%) } & \multicolumn{2}{|c|}{ VPH $-(n=402)$} & \multicolumn{2}{|c|}{$\mathrm{VPH}+(\mathrm{n}=59)$} & \multirow[t]{2}{*}{ RC (IC à 95 \%) } \\
\hline & & & $\%$ & & $\mathbf{n}$ & $\%$ & $\mathbf{n}$ & $\%$ & \\
\hline \multirow[t]{4}{*}{ Origine ethnique } & Autochtone & $18(24,0)$ & $19(33,9)$ & 1,7 (0,7 à 3,8) & 58 & $(14,4)$ & 18 & $(30,5)$ & $3,3(1,7$ à 6,4$)$ \\
\hline & Blanc & $38(50,7)$ & $24(42,9)$ & Référence & 276 & $(68,7)$ & 26 & $(44,1)$ & Référence \\
\hline & Autre & $10(13,3)$ & $3(5,4)$ & $0,5 \quad(0,1$ à 1,9$)$ & 26 & $(6,5)$ & 5 & $(8,5)$ & $2,0 \quad(0,7$ à 5,8$)$ \\
\hline & Non indiquée & $9(12,0)$ & $10(17,9)$ & $1,8 \quad(0,6$ à 5,0) & 42 & $(10,4)$ & 10 & $(16,9)$ & $2,5 \quad(1,1$ à 5,6$)$ \\
\hline \multirow[t]{5}{*}{ Situation financière } & Difficile & $5 \quad(6,7)$ & $5 \quad(8,9)$ & 1,3（0,3 à 4,9） & 19 & $(4,7)$ & 6 & $(10,2)$ & $3,3(1,2$ à 9,4$)$ \\
\hline & Moyenne & $20(26,7)$ & $14(25,0)$ & $0,9 \quad(0,4$ à 2,1$)$ & 110 & $(27,4)$ & 18 & $(30,5)$ & $1,7 \quad(0,9$ à 3,4$)$ \\
\hline & Bonne & $32(42,7)$ & $25(44,6)$ & Référence & 201 & $(50,0)$ & 19 & $(32,2)$ & Référence \\
\hline & Très bonne & $9(12,0)$ & $2 \quad(3,6)$ & $0,3 \quad(0,1$ à 1,4$)$ & 32 & $(8,0)$ & 5 & $(8,5)$ & $1,7 \quad(0,6$ à 4,7) \\
\hline & Non indiquée & $9(12,0)$ & $10(17,9)$ & $1,4 \quad(0,5$ à 4,0) & 40 & $(10,0)$ & 11 & $(18,6)$ & 2,9 (1,3 à 6,5) \\
\hline \multirow[t]{4}{*}{ Niveau de scolarité } & $\begin{array}{l}\text { École secondaire } \\
\text { ou moins }\end{array}$ & $28(37,3)$ & $19(33,9)$ & Référence & 139 & $(34,6)$ & 18 & $(30,5)$ & Référence \\
\hline & Collège & $14(18,7)$ & $8(14,3)$ & $0,8 \quad(0,3$ à 2,4$)$ & 114 & $(28,4)$ & 15 & $(25,4)$ & $1,0 \quad(0,5$ à 2,1$)$ \\
\hline & Université & $25(33,3)$ & $19(33,9)$ & $1,1 \quad(0,5$ à 2,6) & 110 & $(27,4)$ & 16 & $(27,1)$ & $1,1 \quad(0,5$ à 2,3) \\
\hline & Non indiqué & $8(10,7)$ & $10(17,9)$ & 1,8（0,6 à 5,5） & 39 & $(9,7)$ & 10 & $(16,9)$ & $2,0 \quad(0,8$ à 4,6$)$ \\
\hline \multirow[t]{4}{*}{ Usage actuel de tabac } & Oui & $21(28,0)$ & $23(41,1)$ & $2,5(1,1$ à 5,7$)$ & 101 & $(25,1)$ & 24 & $(40,7)$ & $2,5(1,3$ à 5,0$)$ \\
\hline & Ancienne fumeuse & $7 \quad(9,3)$ & $6(10,7)$ & $2,0 \quad(0,6$ à 6,7) & 103 & $(25,6)$ & 11 & $(18,6)$ & $1,1 \quad(0,5$ à 2,6) \\
\hline & N'a jamais fumé & $39(52,0)$ & $17(30,4)$ & Référence & 159 & $(39,6)$ & 15 & $(25,4)$ & Référence \\
\hline & Non indiqué & $8(10,7)$ & $10(17,9)$ & 2,9 (1,0 à 8,5) & 39 & $(9,7)$ & 9 & $(15,3)$ & $2,5 \quad(1,0$ à 6,0$)$ \\
\hline \multirow{4}{*}{$\begin{array}{l}\text { Usage actuel de } \\
\text { contraceptifs oraux }\end{array}$} & Oui & $24(32,0)$ & $15(26,8)$ & $0,7 \quad(0,3$ à 1,6$)$ & 18 & $(4,5)$ & 3 & $(5,1)$ & $1,3 \quad(0,4$ à 4,6) \\
\hline & Non & $31(41,3)$ & $27(48,2)$ & Référence & 292 & $(72,6)$ & 38 & $(64,4)$ & Référence \\
\hline & Ne sait pas & $2 \quad(2,7)$ & $1 \quad(1,8)$ & - & 1 & $(0,2)$ & 1 & $(1,7)$ & - \\
\hline & Non indiqué & $18(24,0)$ & $13(23,2)$ & $0,8 \quad(0,3$ à 2,0$)$ & 91 & $(22,6)$ & 17 & $(28,8)$ & $1,4 \quad(0,8$ à 2,7$)$ \\
\hline \multirow{4}{*}{$\begin{array}{l}\text { Antécédents de } \\
\text { test de Pap }\end{array}$} & Oui & $47(62,7)$ & $41(73,2)$ & Référence & 357 & $(88,8)$ & 46 & $(78,0)$ & Référence \\
\hline & Non & $20(26,7)$ & $5 \quad(8,9)$ & $0,3(0,1$ à 0,8$)$ & 7 & $(1,7)$ & 2 & $(3,4)$ & $2,2(0,4$ à 11,0$)$ \\
\hline & Ne sait pas & $0 \quad(0,0)$ & $0 \quad(0,0)$ & - & 0 & $(0,0)$ & 2 & $(3,4)$ & - \\
\hline & Non indiqués & $8(10,7)$ & $10(17,9)$ & $1,4 \quad(0,5$ à 4,0) & 38 & $(9,5)$ & 9 & $(15,3)$ & $1,8 \quad(0,8$ à 4,0$)$ \\
\hline \multirow[t]{4}{*}{ Antécédents d'ITS } & Oui & $15(20,0)$ & $18(32,1)$ & Référence & 55 & $(13,7)$ & 15 & $(25,4)$ & Référence \\
\hline & Non & $52(69,3)$ & $24(42,9)$ & 0,4 (0,2 à 0,9) & 286 & $(71,1)$ & 29 & $(49,2)$ & $0,4(0,2$ à 0,7$)$ \\
\hline & Ne sait pas & $0 \quad(0,0)$ & $4 \quad(7,1)$ & - & 20 & $(5,0)$ & 6 & $(10,2)$ & - \\
\hline & Non indiqués & $8(10,7)$ & $10(17,9)$ & 1,0 (0,3 à 3,3) & 41 & $(10,2)$ & 9 & $(15,3)$ & $0,8 \quad(0,3$ à 2,0$)$ \\
\hline \multirow[t]{5}{*}{ Nombre d'enfants } & Aucun & $44(58,7)$ & $29(51,8)$ & Référence & 48 & $(11,9)$ & 10 & $(16,9)$ & Référence \\
\hline & 1 & $10(13,3)$ & $9(16,1)$ & $1,4 \quad$ (0,5 à 3,8) & 33 & $(8,2)$ & 10 & $(16,9)$ & $1,5 \quad(0,5$ à 3,9$)$ \\
\hline & 2 & $7 \quad(9,3)$ & $5 \quad(8,9)$ & $1,1 \quad(0,3$ à 3,7$)$ & 113 & $(28,1)$ & 9 & $(15,3)$ & $0,4(0,2$ à 1,0$)$ \\
\hline & 3 et plus & $4 \quad(5,3)$ & $2 \quad(3,6)$ & $0,8 \quad(0,1$ à 4,4) & 167 & $(41,5)$ & 21 & $(35,6)$ & $0,6 \quad(0,3$ à 1,4$)$ \\
\hline & Non indiqué & $10(13,3)$ & $11(19,6)$ & 1,7 (0,6 à 4,4) & 41 & $(10,2)$ & 9 & $(15,3)$ & $1,1 \quad(0,4$ à 2,8$)$ \\
\hline \multirow{5}{*}{$\begin{array}{l}\text { Nombre de partenaires } \\
\text { sexuels au cours de la } \\
\text { dernière année }\end{array}$} & 0 & $7 \quad(9,3)$ & $2 \quad(3,6)$ & $0,7 \quad$ (0,1 à 3,8) & 74 & $(18,4)$ & 11 & $(18,6)$ & $1,4 \quad(0,7$ à 2,9$)$ \\
\hline & $>0^{\mathrm{b}}$ & $0 \quad(0,0)$ & $1 \quad(1,8)$ & - & 7 & $(1,7)$ & 1 & $(1,7)$ & - \\
\hline & 1 & $48(64,0)$ & $19(33,9)$ & Référence & 280 & $(69,7)$ & 30 & $(50,8)$ & Référence \\
\hline & 2 ou plus & $15(20,0)$ & $26(46,4)$ & 4,4 (1,9 à 10,0) & 12 & $(3,0)$ & 11 & $(18,6)$ & $8,6(3,5$ à 21,1$)$ \\
\hline & Non indiqué & $5 \quad(6,7)$ & $8(14,3)$ & 4,0 (1,2 à 13,9) & 29 & $(7,2)$ & 6 & $(10,2)$ & $1,9 \quad(0,7$ à 5,0$)$ \\
\hline Nombre de partenaires & 0 & $6 \quad(8,0)$ & $2 \quad(3,6)$ & $1,0 \quad(0,2$ à 5,6) & 12 & $(3,0)$ & 3 & $(5,1)$ & 3,3 (0,9 à 13,0) \\
\hline sexuels à vie & $>0^{\mathrm{b}}$ & $5 \quad(6,7)$ & $1 \quad(1,8)$ & - & 23 & $(5,7)$ & 4 & $(6,8)$ & - \\
\hline & $1-4$ & $36(48,0)$ & $12(21,4)$ & Référence & 227 & $(56,5)$ & 17 & $(28,8)$ & Référence \\
\hline & $\geq 5$ & $24(32,0)$ & $33(58,9)$ & 4,1 (1,8 à 9,5) & 111 & $(27,6)$ & 28 & $(47,5)$ & $3,4 \quad(1,8$ à 6,4$)$ \\
\hline & Non indiqué & $4 \quad(5,3)$ & $8(14,3)$ & 6,0 (1,5 à 23,5) & 29 & $(7,2)$ & 7 & $(11,9)$ & $3,2(1,2$ à 8,4$)$ \\
\hline Relation anale & Oui & $14(18,7)$ & $10(17,9)$ & $0,9 \quad(0,4$ à 2,4$)$ & 38 & $(9,5)$ & 7 & $(11,9)$ & $0,8 \quad(0,3$ à 1,8$)$ \\
\hline non protégée au cours & Non & $52(69,3)$ & $35(62,5)$ & Référence & 307 & $(76,4)$ & 43 & $(72,9)$ & Référence \\
\hline de la dernière année & Ne sait pas & $0 \quad(0,0)$ & $1 \quad(1,8)$ & - & 3 & $(0,7)$ & 0 & $(0,0)$ & - \\
\hline & Non indiquée & $9(12,0)$ & $10(17,9)$ & $1,6 \quad$ (0,5 à 5,2) & 54 & $(13,4)$ & 9 & $(15,3)$ & $0,9 \quad(0,3$ à 2,6$)$ \\
\hline
\end{tabular}


TABLEAU 1 (Suite)

Résultats d'enquête selon l'âge et le statut à l'égard de l'infection par le VPH

\begin{tabular}{|c|c|c|c|c|c|c|c|c|c|c|c|}
\hline \multirow[t]{3}{*}{ Variables $^{\mathrm{a}}$} & \multirow[t]{3}{*}{ Categories } & \multicolumn{5}{|c|}{ Âge $<30$ ans } & \multicolumn{5}{|c|}{ Âge $\geq 30$ ans } \\
\hline & & \multicolumn{2}{|c|}{ VPH - $(n=75)$} & \multicolumn{2}{|c|}{$\mathrm{VPH}+(\mathrm{n}=56)$} & \multirow[t]{2}{*}{ RC (IC à 95 \%) } & \multicolumn{2}{|c|}{$\mathrm{VPH}-(\mathrm{n}=402)$} & \multicolumn{2}{|c|}{$V P H+(n=59)$} & \multirow[t]{2}{*}{ RC (IC à $95 \%$ ) } \\
\hline & & n & $\%$ & $\mathbf{n}$ & $\%$ & & $\mathbf{n}$ & $\%$ & $\mathbf{n}$ & $\%$ & \\
\hline \multirow[t]{4}{*}{ Relation stable actuellement } & Oui & 54 & $(72,0)$ & 23 & $(41,1)$ & Référence & 289 & $(71,9)$ & 35 & $(59,3)$ & Référence \\
\hline & Non & 11 & $(14,7)$ & 19 & $(33,9)$ & $4,1(1,7$ à 9,9$)$ & 64 & $(15,9)$ & 12 & $(20,3)$ & $1,5 \quad(0,8$ à 3,1$)$ \\
\hline & Pas sûre & 1 & $(1,3)$ & 4 & $(7,1)$ & - & 4 & $(1,0)$ & 3 & $(5,1)$ & - \\
\hline & Non indiquée & 9 & $(12,0)$ & 10 & $(17,9)$ & $2,6 \quad(0,9$ à 7,3$)$ & 45 & $(11,2)$ & 9 & $(15,3)$ & $1,7 \quad(0,7$ à 3,7$)$ \\
\hline \multirow{3}{*}{$\begin{array}{l}\text { Nombre total } \\
\text { de tests de Pap }\end{array}$} & 0 & 22 & $(29,3)$ & 14 & $(25,0)$ & $0,8 \quad(0,3$ à 1,8$)$ & 43 & $(10,7)$ & 11 & $(18,6)$ & $2,1 \quad(1,0$ à 4,5) \\
\hline & $1-4$ & 30 & $(40,0)$ & 25 & $(44,6)$ & Référence & 270 & $(67,2)$ & 33 & $(55,9)$ & Référence \\
\hline & 5 ou + & 23 & $(30,7)$ & 17 & $(30,4)$ & $0,9 \quad(0,4$ à 2,0$)$ & 89 & $(22,1)$ & 15 & $(25,4)$ & $1,4 \quad(0,7$ à 2,7$)$ \\
\hline \multirow{2}{*}{$\begin{array}{l}\text { Nombre total } \\
\text { de colposcopies }\end{array}$} & 0 & 64 & $(85,3)$ & 49 & $(87,5)$ & Référence & 379 & $(94,3)$ & 55 & $(93,2)$ & Référence \\
\hline & $1 \mathrm{ou}+$ & 11 & $(14,7)$ & 7 & $(12,5)$ & $0,8 \quad(0,3$ à 2,3$)$ & 23 & $(5,7)$ & 4 & $(6,8)$ & $1,2 \quad(0,4$ à 3,6$)$ \\
\hline \multirow[t]{3}{*}{ Pire résultat cytologique ${ }^{c}$} & Aucun antécédent & 22 & $(29,3)$ & 14 & $(25,0)$ & $1,0 \quad(0,5$ à 2,4$)$ & 43 & $(10,7)$ & 11 & $(18,6)$ & $2,0(1,0$ à 4,3$)$ \\
\hline & Normal & 42 & $(56,0)$ & 26 & $(46,4)$ & Référence & 323 & $(80,3)$ & 40 & $(67,8)$ & Référence \\
\hline & Autre que normal & 11 & $(14,7)$ & 16 & $(28,6)$ & $2,4 \quad(0,9$ à 5,8$)$ & 36 & $(9,0)$ & 8 & $(13,6)$ & $1,8 \quad(0,8$ à 4,1$)$ \\
\hline \multirow[t]{3}{*}{ Pire résultat histologique ${ }^{c}$} & Aucun antécédent & 64 & $(85,3)$ & 49 & $(87,5)$ & Référence & 379 & $(94,3)$ & 55 & $(93,2)$ & Référence \\
\hline & Normal & 3 & $(4,0)$ & 2 & $(3,6)$ & $0,9 \quad(0,1$ à 5,4$)$ & 12 & $(3,0)$ & 1 & $(1,7)$ & $0,6 \quad(0,1$ à 4,5$)$ \\
\hline & Autre que normal & 8 & $(10,7)$ & 5 & $(8,9)$ & $0,8(0,3$ à 2,7$)$ & 11 & $(2,7)$ & 3 & $(5,1)$ & $1,9 \quad(0,5$ à 6,9$)$ \\
\hline
\end{tabular}

Abréviations : ASC H, cellules malpighiennes atypiques - on ne peut écarter une lésion de haut grade; ASC US, atypie des cellules malpighiennes de signification indéterminée; HSIL, lésions malpighiennes intra épithéliales de haut grade; IC, intervalle de confiance; ITS, infection transmise sexuellement; LSIL, lésions malpighiennes intra épithéliales de bas grade; NIC, néoplasie intra épithéliale cervicale; Pap, Papanicolaou; RC, rapport de cotes; VPH, virus du papillome humain; VPH- , négative pour le VPH; VPH+, positive pour le VPH.

Remarque : Les valeurs en caractères gras sont significatives.

${ }^{a}$ Ces variables sont toutes auto-déclarées.

${ }^{b}$ Valeur obtenue en combinant l'information sur le nombre d'enfants et les questions sur l'activité sexuelle.

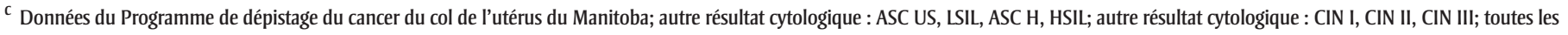
autres variables sont auto déclarées par les participantes.

TABLEAU 2

Distribution par âge des femmes selon le statut infectieux et le type de VPH (échelle individuelle)

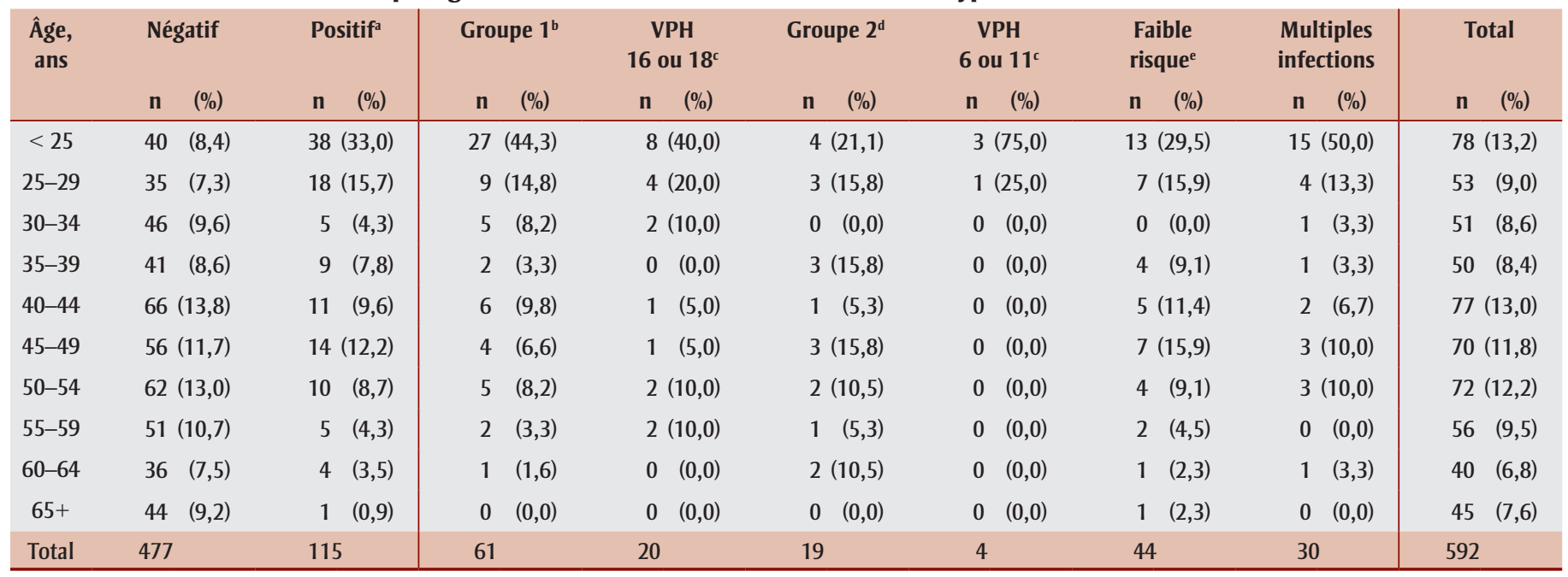

Abréviations : VPH, virus du papillome humain.

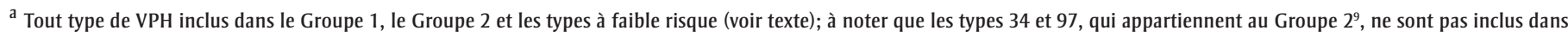
les types de VPH pris en compte dans la méthodologie utilisée pour la présente étude.

${ }^{b}$ Groupe 1 : types 16, 18, 31, 33, 35, 39, 45, 51, 52, 56, 58, 59 du VPH.

c Un type ou les deux types sont présents en même temps.

${ }^{\mathrm{d}}$ Groupe 2 : types 26, 53, 66, 67, 68, 70, 73, 82, 30, 69, 85 du VPH.

e Faible risque : types 6, 11, 13, 32, 40, 42, 43, 44, 54, 61, 62, 71, 72, 74, 81, 83, 84, 86, 87, 89, 90, 91 du VPH. 
TABLEAU 3

Prévalence individuelle du VPH selon le résultat cytologique

\begin{tabular}{|c|c|c|c|c|c|c|c|c|c|}
\hline \multirow{2}{*}{$\begin{array}{l}\text { Types de } \\
\text { VPH }\end{array}$} & \multirow{2}{*}{$\begin{array}{c}\text { Manquant } \\
\text { n }\end{array}$} & \multirow{2}{*}{$\begin{array}{c}\text { Normal } \\
\text { n }\end{array}$} & \multirow{2}{*}{$\begin{array}{c}\text { Insatisfaisant } \\
\text { n }\end{array}$} & \multirow{2}{*}{$\begin{array}{c}\text { ASC-US } \\
\mathbf{n}\end{array}$} & \multirow{2}{*}{$\begin{array}{l}\text { LSIL } \\
\mathbf{n}\end{array}$} & \multirow{2}{*}{$\begin{array}{c}\text { ASC-H } \\
n\end{array}$} & \multirow{2}{*}{$\begin{array}{c}\text { HSIL } \\
\text { n }\end{array}$} & \multicolumn{2}{|c|}{ Total } \\
\hline & & & & & & & & $\mathbf{n}$ & $\%$ \\
\hline Négatif & 11 & 428 & 16 & 13 & 6 & 2 & 1 & 477 & 80,6 \\
\hline N'importe lequel ${ }^{\mathrm{a}}$ & 5 & 89 & 2 & 6 & 10 & 0 & 3 & 115 & 19,4 \\
\hline 6 ou $11^{b}$ & 0 & 3 & 1 & 0 & 0 & 0 & 0 & 4 & 0,7 \\
\hline 16 & 2 & 10 & 0 & 1 & 2 & 0 & 0 & 15 & 2,5 \\
\hline 16 ou $18^{b}$ & 2 & 14 & 0 & 1 & 2 & 0 & 1 & 20 & 3,4 \\
\hline Groupe $1^{c}$ & 3 & 46 & 1 & 2 & 6 & 0 & 3 & 61 & 10,3 \\
\hline Groupe $2^{d}$ & 1 & 13 & 0 & 2 & 2 & 0 & 1 & 19 & 3,2 \\
\hline Faible risque ${ }^{\mathrm{e}}$ & 1 & 36 & 1 & 3 & 3 & 0 & 0 & 44 & 7,4 \\
\hline Multiples ${ }^{f}$ & 1 & 22 & 0 & 2 & 4 & 0 & 1 & 30 & 5,1 \\
\hline Total & 16 & 517 & 18 & 19 & 16 & 2 & 4 & 592 & \\
\hline
\end{tabular}

Abréviations : ASC H, cellules malpighiennes atypiques - on ne peut écarter une lésion de haut grade; ASC US, atypie des cellules malpighiennes de signification indéterminée; HSIL, lésions malpighiennes intra épithéliales de haut grade; LSIL, lésions malpighiennes intra épithéliales de bas grade; VPH, virus du papillome humain;.

${ }^{\mathrm{a}}$ Tout type de VPH inclus dans le Groupe 1, le Groupe 2 et les types à faible risque (voir texte suivant); à noter que les types 34 et 97 , qui appartiennent au Groupe $2^{9}$, ne sont pas inclus dans les types de VPH pris en compte dans la méthodologie utilisée pour la présente étude.

b Un type ou l'autre ou les deux peuvent être présents en même temps.

${ }^{c}$ Groupe 1 : types 16, 18, 31, 33, 35, 39, 45, 51, 52, 56, 58, 59 du VPH.

${ }^{\mathrm{d}}$ Groupe 2 : types 26, 53, 66, 67, 68, 70, 73, 82, 30, 69, $85 \mathrm{du} \mathrm{VPH.}$

e Faible risque : types 6, 11, 13, 32, 40, 42, 43, 44, 54, 61, 62, 71, 72, 74, 81, 83, 84, 86, 87, 89, 90, 91 du VPH.

${ }^{\mathrm{f}}$ Multiples infections à VPH.

de bas grade histologique (LSIL; 10/16) et $75 \%$ des cas de lésion malpighienne intra-épithéliale de haut grade histologique (HSIL; 3/4). Un type de VPH du Groupe 1 a été détecté chez $6 \%$ des répondantes ayant obtenu un résultat insatisfaisant au test de Pap (1/18), $11 \%$ des cas d'ASC-US (2/19), $38 \%$ des cas de LSIL (6/16) et $75 \%$ des cas de HSIL (3/4). Les types de VPH du Groupe 1 (dans l'ensemble : 10,3 \% [61/592]; chez les participantes infectées par le VPH : 53,0 \% [61/115]) ont été détectés plus fréquemment que ceux du Groupe 2 (dans l'ensemble : $3,2 \%$ [19/592]; chez les participantes infectées par le VPH : 16,5 \% [19/115]) et que les types de VPH à faible risque (dans l'ensemble : 7,4 \% [44/592]; chez les participantes infectées par le VPH : 38,2 \% [44/115]). Les résultats au test de Pap n'étaient pas disponibles pour $3 \%$ (16/592) des échantillons de VPH testés.

Nous ignorons pourquoi certains tests de Pap n'ont pas été envoyés au laboratoire pour une évaluation. Il se peut que les cliniciens qui ont effectué ces tests aient pensé que leur seule tâche pour cette étude consistait à prélever un échantillon de tissu pour le typage du VPH et qu'ils n'ont pas demandé de test cytologique courant.
Parmi les types du Groupe 1, le VPH-16 était celui qui était le plus souvent décelé (10\%), suivi des types 39 (5\%), 58 (5\%), 18 (4\%), 35 (4\%), 51 (4\%), 52 (4\%), $59(4 \%)$ et $33(3 \%)$ (tableau 4). À l'intérieur du genre alpha, les espèces 9 (29 \%; 45/157), 3 (19\%; 29/157) et 7 (17\%; 26/157) ont été les plus fréquemment détectées. L'espèce 9 inclut des virus apparentés au VPH-16, alors que l'espèce 7 englobe ceux apparentés au VPH-18, et l'espèce 3 comprend des types de VPH à faible risque.

\section{Discussion}

Il est difficile de comparer la prévalence des infections par le VPH entre les études en raison des différences fréquentes dans les techniques de typage, les populations échantillonnées et les stratégies d'échantillonnage. En outre, les taux de prévalence sont rarement standardisés selon l'âge. Cela dit, une méta-analyse a fait état de taux d'infection par le VPH variant entre 7 et $8 \%$ en Europe et en Asie, atteignant $14 \%$ en Amérique du Nord et $23 \%$ en Afrique chez les femmes ayant obtenu des résultats cytologiques normaux $^{20}$. Aux États-Unis, les taux ont été estimés à $27 \%$ chez les femmes de 14 à $59 \mathrm{ans}^{21}$. Notre étude a déterminé un taux de prévalence du VPH de 19 \% (17 \% chez celles qui avaient obtenu un résultat normal au test de Pap). Le VPH-16 était le type le plus souvent détecté au niveau du col, les autres types à risque élevé courants étant les types 18, 33, 35, 39, 51, 52, 58 et 59 . Ces résultats concordent avec d'autres observations montrant que les types 16, $18,31,39,51,52,56$ et 58 du VPH sont parmi les plus fréquents dans le monde chez les femmes ayant obtenu des résultats cytologiques normaux ${ }^{22}$; les types 16,18 , $31,33,45,51,52,56$ et 58 de VPH étant plus fréquents chez les femmes ayant reçu un diagnostic de lésions cervicales de bas grade $^{23}$ alors que les types $16,18,31,33,35$, 45, 52 et 58 de VPH étaient plus fréquents chez les femmes ayant reçu un diagnostic d'anomalies de haut grade ${ }^{24}$. Le classement des taux de prévalence selon le type de VPH varie cependant selon la région et selon le pays $^{22-24}$. Par exemple, une étude de population en Belgique a indiqué que le type de VPH à risque élevé le plus répandu était le VPH-16 (3,7\%), suivi des types 31,51 et 53 , qui ont été détectés dans au moins $2 \%$ de la population (le VPH-18 a été retrouvé dans $1,5 \%$ de la population) $)^{25}$. Une étude basée sur une population en Suède a relevé un taux de prévalence de $2,5 \%$ pour le VPH-16, de 1,4\% pour le $\mathrm{VPH}-31$, de $0,9 \%$ pour le $\mathrm{VPH}-45$ et 
TABLEAU 4

Prévalence des infections dues à une espèce génitale de VPH du genre alpha

\begin{tabular}{|c|c|c|c|c|c|c|c|c|c|}
\hline \multirow[t]{2}{*}{ Espèce } & \multirow{2}{*}{$\begin{array}{c}\text { Type } \\
\text { de VPH }\end{array}$} & \multirow{2}{*}{$\begin{array}{c}\text { Manquant } \\
\text { n }\end{array}$} & \multirow{2}{*}{$\begin{array}{c}\text { Négatif } \\
\mathbf{n}\end{array}$} & \multirow{2}{*}{$\begin{array}{c}\text { Insatisfaisant } \\
\text { n }\end{array}$} & \multirow{2}{*}{$\begin{array}{c}\text { ASC-US } \\
n\end{array}$} & \multirow{2}{*}{$\begin{array}{l}\text { LSIL } \\
\text { n }\end{array}$} & \multirow{2}{*}{$\begin{array}{c}\text { HSIL } \\
n\end{array}$} & \multicolumn{2}{|c|}{ Total } \\
\hline & & & & & & & & $\mathbf{n}$ & $\%$ \\
\hline \multirow[t]{3}{*}{ A1 } & 32 & 0 & 4 & 0 & 0 & 0 & 0 & 4 & 2,5 \\
\hline & 42 & 0 & 6 & 0 & 0 & 0 & 0 & 6 & 3,8 \\
\hline & Total & 0 & 10 & 0 & 0 & 0 & 0 & 10 & 6,4 \\
\hline \multirow[t]{8}{*}{ A3 } & 62 & 0 & 5 & 0 & 1 & 0 & 0 & 6 & 3,8 \\
\hline & 72 & 0 & 2 & 0 & 0 & 0 & 0 & 2 & 1,3 \\
\hline & 81 & 0 & 5 & 0 & 0 & 1 & 0 & 6 & 3,8 \\
\hline & 83 & 0 & 3 & 0 & 0 & 1 & 0 & 4 & 2,5 \\
\hline & 84 & 0 & 2 & 0 & 0 & 0 & 0 & 2 & 1,3 \\
\hline & 86 & 0 & 2 & 0 & 0 & 0 & 0 & 2 & 1,3 \\
\hline & 89 & 0 & 6 & 0 & 0 & 1 & 0 & 7 & 4,5 \\
\hline & Total & 0 & 25 & 0 & 1 & 3 & 0 & 29 & 18,5 \\
\hline \multirow[t]{4}{*}{ A5 } & 51 & 0 & 4 & 0 & 0 & 1 & 1 & 6 & 3,8 \\
\hline & 69 & 0 & 1 & 0 & 0 & 0 & 0 & 1 & 0,6 \\
\hline & 82 & 0 & 1 & 0 & 0 & 0 & 0 & 1 & 0,6 \\
\hline & Total & 0 & 6 & 0 & 0 & 1 & 1 & 8 & 5,1 \\
\hline \multirow[t]{5}{*}{ A6 } & 30 & 0 & 2 & 0 & 0 & 1 & 0 & 3 & 1,9 \\
\hline & 53 & 0 & 0 & 0 & 0 & 1 & 1 & 2 & 1,3 \\
\hline & 56 & 0 & 3 & 0 & 0 & 0 & 0 & 3 & 1,9 \\
\hline & 66 & 0 & 4 & 0 & 0 & 0 & 0 & 4 & 2,5 \\
\hline & Total & 0 & 9 & 0 & 0 & 2 & 1 & 12 & 7,6 \\
\hline \multirow[t]{7}{*}{ A7 } & 18 & 0 & 5 & 0 & 0 & 0 & 1 & 6 & 3,8 \\
\hline & 39 & 1 & 4 & 0 & 0 & 1 & 1 & 7 & 4,5 \\
\hline & 45 & 0 & 1 & 0 & 0 & 0 & 0 & 1 & 0,6 \\
\hline & 59 & 0 & 5 & 0 & 0 & 1 & 0 & 6 & 3,8 \\
\hline & 70 & 0 & 4 & 0 & 1 & 0 & 0 & 5 & 3,2 \\
\hline & 85 & 0 & 1 & 0 & 0 & 0 & 0 & 1 & 0,6 \\
\hline & Total & 1 & 20 & 0 & 1 & 2 & 2 & 26 & 16,6 \\
\hline \multirow[t]{4}{*}{ A8 } & 7 & 0 & 1 & 0 & 0 & 0 & 0 & 1 & 0,6 \\
\hline & 40 & 0 & 1 & 0 & 2 & 0 & 0 & 3 & 1,9 \\
\hline & 91 & 0 & 0 & 0 & 1 & 0 & 0 & 1 & 0,6 \\
\hline & Total & 0 & 2 & 0 & 3 & 0 & 0 & 5 & 3,2 \\
\hline \multirow[t]{8}{*}{ A9 } & 16 & 2 & 10 & 0 & 1 & 2 & 0 & 15 & 9,6 \\
\hline & 31 & 0 & 2 & 0 & 1 & 0 & 0 & 3 & 1,9 \\
\hline & 33 & 0 & 5 & 0 & 0 & 0 & 0 & 5 & 3,2 \\
\hline & 35 & 0 & 4 & 0 & 0 & 1 & 1 & 6 & 3,8 \\
\hline & 52 & 0 & 6 & 0 & 0 & 0 & 0 & 6 & 3,8 \\
\hline & 58 & 1 & 4 & 1 & 0 & 1 & 0 & 7 & 4,5 \\
\hline & 67 & 1 & 1 & 0 & 0 & 1 & 0 & 3 & 1,9 \\
\hline & Total & 4 & 32 & 1 & 2 & 5 & 1 & 45 & 28,7 \\
\hline \multirow[t]{5}{*}{ A10 } & 6 & 0 & 2 & 0 & 0 & 0 & 0 & 2 & 1,3 \\
\hline & 11 & 0 & 1 & 1 & 0 & 0 & 0 & 2 & 1,3 \\
\hline & 44 & 1 & 3 & 0 & 1 & 0 & 0 & 5 & 3,2 \\
\hline & 74 & 0 & 5 & 0 & 0 & 0 & 0 & 5 & 3,2 \\
\hline & Total & 1 & 11 & 1 & 1 & 0 & 0 & 14 & 8,9 \\
\hline A11 & 73 & 0 & 0 & 0 & 1 & 0 & 0 & 1 & 0,6 \\
\hline A13 & 54 & 0 & 4 & 0 & 0 & 1 & 0 & 5 & 3,2 \\
\hline \multirow[t]{3}{*}{ Autre } & 8 & 0 & 1 & 0 & 0 & 0 & 0 & 1 & 0,6 \\
\hline & 38 & 0 & 0 & 0 & 0 & 1 & 0 & 1 & 0,6 \\
\hline & Total & 0 & 1 & 0 & 0 & 1 & 0 & 2 & 1,3 \\
\hline Total & & 6 & 120 & 2 & 9 & 15 & 5 & 157 & \\
\hline
\end{tabular}

Abréviations : ASC H, cellules malpighiennes atypiques - on ne peut écarter une lésion de haut grade; ASC US, atypie des cellules malpighiennes de signification indéterminée; HSIL, lésions malpighiennes intra épithéliales de haut grade; LSIL, lésions malpighiennes intra épithéliales de bas grade; VPH, virus du papillome humain. de $0,7 \%$ pour le VPH-18; $13,0 \%$ des femmes présentaient des infections multiples $^{26}$. De nombreuses études ont signalé une augmentation des infections à VPH chez les femmes de 60 ans et plus ${ }^{27}$. Le nombre de cas n'était pas suffisant pour qu'on puisse confirmer cette tendance au Manitoba.

Quelques études ont examiné la prévalence du VPH au Canada. Une étude menée en Colombie-Britannique a relevé un taux global de prévalence du VPH de 16,8 \% (VPH à risque élevé : 13,9\%; VPH-16 : $10,7 \%)^{28}$; une étude ontarienne a fait état d'un taux global d'infection de 13,3 \% (VPH à risque élevé : 9,6 \%; VPH-16 : $7,3 \%{ }^{29}$; une étude effectuée au NouveauBrunswick a estimé la prévalence à $28 \%$ $(\mathrm{VPH} \text { à risque élevé : } 21 \%)^{30}$. Dans le cadre d'une étude réalisée entre 1992 et 1995 , on a recruté une forte proportion de femmes autochtones (42\%) fréquentant une clinique située dans un quartier défavorisé du centre-ville de Winnipeg (Manitoba) et on a constaté que les taux d'infection par le VPH chez les femmes autochtones et non autochtones étaient comparables (respectivement 33,6\% et $31,8 \%)^{31}$. À cause des différences dans les populations incluses, il est toutefois difficile de comparer les résultats de notre étude avec ceux de cette étude antérieure.

Ce que montrent invariablement les études, c'est que plus les lésions sont graves, plus la prévalence du VPH à risque élevé augmente. Par exemple, une méta-analyse a obtenu un taux de VPH à risque élevé de $71,9 \%$ (IC à $95 \%$ : $62,8 \%$ à $80,9 \%$ ) chez les cas de LSIL ${ }^{23}$ et de $88,3 \%$ (IC à $95 \%: 85,8 \%$ à 90,8\%) chez les cas de HSIL ${ }^{24}$. Moore et collab. ${ }^{28}$ ont observé qu'on retrouvait des VPH à risque élevé dans $52,3 \%$ des LSIL et 79,4 \% des HSIL. Ils ont également constaté que la positivité à l'égard du VPH augmentait, allant de $12,3 \%$ pour les résultats normaux à $81,0 \%$ pour les lésions de haut grade ${ }^{28}$, en passant par 19,6\% pour les lésions bénignes et 69,3\% pour les lésions de bas grade. Nous avons observé dans notre étude que 37,5 \% des LSIL étaient positives pour le VPH à risque élevé (Groupe 1) et $75 \%$ parmi les HSIL. 
Un certain nombre de cofacteurs sont associés au risque d'infection par le VPH et à différents grades d'anomalies cervicales, bon nombre d'entre eux étant liés aux comportements sexuels. Les facteurs qui ont été le plus régulièrement associés à des taux accrus d'infection par le VPH sont les suivants : plus jeune âge et plus grand nombre de partenaires sexuels récents et à $v^{3} e^{32,33}$. D'autres cofacteurs d'infection par le VPH ont également été signalés, mais pas systématiquement : âge lors de la première relation sexuelle, usage de tabac, usage de contraceptifs oraux, origine ethnique, consommation d'alcool, antécédents d'ITS, revenu et utilisation du condom $^{33-41}$. L'analyse multidimensionnelle a révélé que l'âge, l'origine ethnique et le nombre de partenaires sexuels au cours de l'année écoulée constituaient des prédicteurs indépendants. Notre étude laisse également penser que certains de ces facteurs de risque sont communs à tous les groupes d'âge alors que d'autres facteurs ne se retrouvent que chez les femmes soit plus jeunes, soit plus âgées.

Les femmes qui n'avaient aucun antécédent de dépistage de cancer du col de l'utérus et celles qui n'avaient pas subi suffisamment de tests de dépistage présentaient des taux plus élevés d'incidence du cancer du col de l'utérus que les femmes qui faisaient régulièrement l'objet d'un dépistage $\mathrm{e}^{42-45}$. Dans cette étude, les femmes de 30 ans et plus qui n'avaient jamais subi de test de Pap étaient plus nombreuses à être infectées par le VPH.

\section{Limites de l'étude}

Cette étude comporte plusieurs limites. Comme presque toutes les études de séroprévalence, notre étude s'appuyait sur des échantillons opportunistes et n’était pas basée sur une population. Les résultats ne représentent donc pas nécessairement le taux d'infection par le VPH dans la population féminine en général. La publicité entourant la Semaine manitobaine du test de Pap et le dépistage effectué durant une journée par des cliniques pourraient également créer un biais de sélection en encourageant les femmes qui tardent à passer le test de Pap à finalement se faire tester. Il est difficile de prédire la conséquence d'un tel biais sur l'analyse des facteurs de risque, mais si le biais est différentiel, cela peut expliquer pourquoi le risque d'infection était plus élevé dans certains groupes. Le taux de participation au dépistage du cancer du col de l'utérus au Manitoba entre 2007 et 2009 atteignait $65,9 \%$ chez les femmes de 20 à 69 ans. Leurs résultats cytologiques variaient de la façon suivante : cytologie normale, 95,5\%; ASC-US, 3,1 \%; LSIL, 2,1 \%; cellules glandulaires atypiques (AGC), 0,1 \%; ASC-H, 0,3 \%; HSIL, 0,9 \%. Parmi les participantes à l'étude, le taux de participation au dépistage du cancer du col depuis 2001 s'établissait à 84,8\% (502/592), les résultats de leur cytologie se répartissant de la façon suivante : cytologie normale, $87 \%(517 / 592)$; ASC-US, $3 \%(19 / 592)$; LSIL, $3 \%$ (16/592); ASC-H, 0,3 \% (2/592); HSIL, 1 \% (4/592).

Cette comparaison semble indiquer que la plupart des participantes à l'étude ont subi régulièrement un dépistage du cancer du col utérin et que leurs résultats cytologiques étaient comparables à ceux des femmes qui avaient participé au dépistage du cancer du col utérin au Manitoba entre 2007 et 2009. Le fait que l'étude soit de type transversal ne permet pas d'établir une relation causale entre l'infection par le VPH et les cofacteurs étudiés. De plus, les questionnaires autoadministrés peuvent être sujets à des biais. Les observations concordent néanmoins avec notre connaissance actuelle des facteurs de risque d'infection par le VPH. En raison de la grande sensibilité de la méthode de détection du VPH, la signification clinique de la présente étude est limitée. L'amplification par PCR permet de détecter même une seule copie des gènes ciblés (ADN de L1), et cette sensibilité ne se traduit pas nécessairement par une infection de signification clinique. Depuydt et collab. ont montré qu'en deçà d'une charge virale critique, il est très rare qu'on détecte des lésions visuellement décelables ${ }^{46}$. Un test très sensible offre la possibilité de limiter le triage des personnes infectées par le VPH.

\section{Conclusion}

Comme l'indiquent les résultats de notre étude, la distribution des types de VPH oncogènes au Manitoba concorde avec celle qui a été signalée au Canada et dans d'autres pays. Nos données fournissent des renseignements de base sur la prévalence du VPH dans une population non vaccinée au Manitoba. En outre, le couplage de données permet de valider le principe de l'applicabilité du couplage avec un registre basé sur une population pour évaluer les programmes de vaccination contre le VPH dans les provinces ou territoires capables d'effectuer un tel couplage.

\section{Références}

1. Garnett GP, Kim JJ, French K, Goldie SJ. Chapter 21: Modelling the impact of HPV vaccines on cervical cancer and screening programmes. Vaccine. 2006;24 Suppl 3:S178-86.

2. Brisson M, Van de Velde N, Boily M-C. Different population-level vaccination effectiveness for HPV types 16, 18, 6 and 11 . Sex Transm Infect. 2011;87(1):41-3.

3. Smith MA, Canfell K, Brotherton JML, Lew J-B, Barnabas RV. The predicted impact of vaccination on human papillomavirus infections in Australia. Int $\mathrm{J}$ Cancer. 2008;123(8):1854-63.

4. Donovan B, Franklin N, Guy R, Grulich AE, Regan DG, Ali H, et collab. Quadrivalent human papillomavirus vaccination and trends in genital warts in Australia: analysis of national sentinel surveillance data. Lancet Infect Dis. 2011;11(1):39-44.

5. Fairley CK, Hocking JS, Gurrin LC, Chen MY, Donovan B, Bradshaw CS. Rapid decline in presentations of genital warts after the implementation of a national quadrivalent human papillomavirus vaccination programme for young women. Sex Transm Infect. 2009;85(7):499-502.

6. Muñoz N, Kjaer SK, Sigurdsson K, Iversen OE, Hernandez-Avila $\mathrm{M}$, Wheeler $\mathrm{CM}$, et collab. Impact of human papillomavirus (HPV)-6/11/16/18 vaccine on all HPVassociated genital diseases in young women. J Natl Cancer Inst. 2010;102(5):325-39.

7. Tota J, Mahmud SM, Ferenczy A, Coutlée F, Franco EL. Promising strategies for cervical cancer screening in the post-human papillomavirus vaccination era. Sex Health. 2010;7(3):376-82. 
8. Kliewer EV, Demers AA, Brisson $\mathrm{M}$, Severini A, Lotocki R, Elias B, et collab. Le système manitobain de surveillance et d'évaluation du vaccin contre le virus du papillome humain. Rapports sur la santé. 2010;21(2):1-8.

9. Bouvard V, Baan R, Straif K, Grosse Y, Secretan B, El Ghissassi F, et collab. A review of human carcinogens-Part B: biological agents. Lancet Oncol. 2009;10(4):321-2.

10. Higuchi R. Simple and rapid preparation of samples for PCR. Dans : Erlich HA (dir). PCR Technology: Principles and Applications for DNA Amplification. New York: Stockton Press; 1989. p. 31-8.

11. Josefsson A, Magnusson P, Gyllensten U. Human papillomavirus detection by PCR and typing by Dot-Blot. Dans : Peeling RW, Sparling PF (dir.). Sexually Transmitted Diseases. Methods and Protocols. Totowa (NJ): Humana Press; 1999. p. 171-93.

12. Gravitt PE, Peyton CL, Alessi TQ, Wheeler CM, Coutlée F, Hildesheim A, et collab. Improved amplification of genital human papillomaviruses. J Clin Microbiol. 2000;38(1):357-61.

13. de Roda Husman AM, Walboomers JM, van den Brule AJ, Meijer CJ, Snijders PJ. The use of general primers GP5 and GP6 elongated at their 3' ends with adjacent highly conserved sequences improves human papillomavirus detection by PCR. J. Gen. Virol. 1995 Apr;76(Pt 4):1057-62.

14. Evander M, Edlund K, Bodén E, Gustafsson A, Jonsson M, Karlsson R, et collab. Comparison of a one-step and a two-step polymerase chain reaction with degenerate general primers in a population-based study of human papillomavirus infection in young Swedish women. J Clin Microbiol. 1992;30(4):987-92.

15. Strauss S, Jordens JZ, Desselberger U, Gray JJ. Single-tube real-time nested polymerase chain reaction for detecting human papillomavirus DNA. Diagn Mol Pathol. 2000;9(3):151-7.
16. Bulkmans NW, Berkhof J, Rozendaal L, van Kemenade FJ, Boeke AJ, Bulk S, et collab. Human papillomavirus DNA testing for the detection of cervical intraepithelial neoplasia grade 3 and cancer: 5-year follow-up of a randomised controlled implementation trial. Lancet. 2007;370(9601):1764-72.

17. Zubach V, Smart G, Ratnam S, Severini A. Novel microsphere-based method for detection and typing of 46 mucosal human papillomavirus types. J Clin Microbiol. 2012;50(2):460-4.

18. Rebbapragada A, Perusini A, Di Prima A, Salit I, Goleski V, Severini A. Evaluation of test performance characteristics and operational parameters of $5 \mathrm{HPV}$ genotyping assays. 26th International Papillomavirus Conference; 3-8 juillet 2010; Montréal, Canada.

19. de Villiers EM, Fauquet C, Broker TR, Bernard HU, zur Hausen $\mathrm{H}$. Classification of papillomaviruses. Virology. 2004;324(1):17-27.

20. de Sanjosé S, Diaz M, Castellsagué X, Clifford G, Bruni L, Muñoz N, et collab. Worldwide prevalence and genotype distribution of cervical human papillomavirus DNA in women with normal cytology: a meta-analysis. Lancet Infect Dis. 2007;7 (7):453-9.

21. Dunne EF, Unger ER, Sternberg $M$, McQuillan G, Swan DC, Patel SS, et collab. Prevalence of HPV infection among females in the United States. JAMA. 2007;297(8):813-9.

22. Bruni L, Diaz M, Castellsagué X, Ferrer E, Bosch FX, de Sanjosé S. Cervical human papillomavirus prevalence in 5 continents: meta-analysis of 1 million women with normal cytological findings. J Infect Dis. 2010;202(12):1789-99.

23. Clifford GM, Rana RK, Franceschi S, Smith JS, Gough G, Pimenta JM. Human papillomavirus genotype distribution in low-grade cervical lesions: comparison by geographic region and with cervical cancer. Cancer Epidemiol Biomarkers Prev. 2005;14(5):1157-64.
24. Smith JS, Lindsay L, Hoots B, Keys J, Franceschi S, Winer R, et collab. Human papillomavirus type distribution in invasive cervical cancer and high-grade cervical lesions: a meta-analysis update. Int J Cancer. 2007;121(3):621-32.

25. Arbyn M, Sasieni P, Meijer CJ, Clavel C, Koliopoulos G, Dillner J. Chapter 9: Clinical applications of HPV testing: a summary of meta-analyses. Vaccine. 2006;24(Suppl. 3):S78-S89.

26. Naucler P, Ryd W, Törnberg S, Strand A, Wadell G, Hansson BG, et collab. HPV type-specific risks of high-grade CIN during 4 years of follow-up: a population-based prospective study. $\mathrm{Br} \mathrm{J}$ Cancer. 2007;97(1):129-32.

27. Burchell AN, Winer RL, de Sanjosé S, Franco EL. Chapter 6: Epidemiology and transmission dynamics of genital HPV infection. Vaccine. 2006;24(Suppl. 3):S52-S61.

28. Moore RA, Ogilvie G, Fornika D, Moravan V, Brisson M, Amirabbasi-Beik M, et collab. Prevalence and type distribution of human papillomavirus in 5,000 British Columbia women-implications for vaccination. Cancer Causes Control. 2009;20(8):1387-96.

29. Sellors JW, Mahony JB, Kaczorowski J, Lytwyn A, Bangura H, Chong S, et collab. Prevalence and predictors of human papillomavirus infection in women in Ontario, Canada. Survey of HPV in Ontario Women (SHOW) Group. CMAJ. 2000;163(5):503-8.

30. Kurz J, Mitra K, Adam R, Miao X, MacKay JS, Isa NN, et collab. PCR detection and typing of genital papillomavirus in a New Brunswick population. Int J Cancer. 1993;55(4):604-8.

31. Young TK, McNicol P, Beauvais J. Factors associated with human papillomavirus infection detected by polymerase chain reaction among urban Canadian aboriginal and non-aboriginal women. Sex Transm Dis. 1997;24(5):293-8.

32. Trottier H, Franco EL. The epidemiology of genital human papillomavirus infection. Vaccine. 2006;24(Suppl. 1):S1-S15. 
33. Scheurer ME, Tortolero-Luna G, Adler-Storthz K. Human papillomavirus infection: biology, epidemiology, and prevention. Int $\mathrm{J}$ Gynecol Cancer. 2005;15(5):727-46.

34. Moscicki AB, Schiffman M, Kjaer S, Villa LL. Chapter 5: Updating the natural history of HPV and anogenital cancer. Vaccine. 2006;24(Suppl. 3):S42-S51.

35. Trottier H, Burchell AN. Epidemiology of mucosal human papillomavirus infection and associated diseases. Public Health Genomics. 2009;12(5-6):291-307.

36. Winer RL, Lee SK, Hughes JP, Adam DE, Kiviat NB, Koutsky LA. Genital human papillomavirus infection: incidence and risk factors in a cohort of female university students. Am J Epidemiol. 2003;157(3):218-26.

37. Ho GYF, Bierman R, Beardsley L, Chang CJ, Burk RD. Natural history of cervicovaginal papillomavirus infection in young women. N Engl J Med. 1998;338(7):423-8.

38. Moscicki AB, Hills N, Shiboski S, Powell K, Jay N, Hanson E, et collab. Risks for incident human papillomavirus infection and low-grade squamous intraepithelial lesion development in young females. JAMA. 2001;285(23):2995-3002.

39. Goodman MT, Shvetsov YB, McDuffie K, Wilkens LR, Zhu X, Thompson PJ, et collab. Prevalence, acquisition, and clearance of cervical human papillomavirus infection among women with normal cytology: Hawaii Human Papillomavirus Cohort Study. Cancer Res. 2008;68(21):8813-24.

40. Giuliano AR, Harris R, Sedjo RL, Baldwin S, Roe D, Papenfuss MR, et collab. Incidence, prevalence, and clearance of type-specific human papillomavirus infections: The Young Women's Health Study. J Infect Dis. 2002;186(4):462-9.

41. Sellors JW, Karwalajtys TL, Kaczorowski J, Mahony JB, Lytwyn A, Chong S, et collab. Incidence, clearance and predictors of human papillomavirus infection in women. CMAJ. 2003;168(4):421-5.
42. Decker K, Demers A, Chateau D, Musto G, Nugent Z, Lotocki R, et collab. Papanicolaou test utilization and frequency of screening opportunities among women diagnosed with cervical cancer. Open Med. 2009;3(3):140-7.

43. Boulanger JC, Fauvet $R$, Urrutiaguer $S$, Drean Y, Sevestre H, Ganry O, et collab. Histoire cytologique des cancers du col utérin diagnostiqués en France en 2006. Gynecol Obstet Fertil. 2007;35(9):764-71.

44. van der Aa MA, Schutter EM, LooijenSalamon M, Martens JE, Siesling S. Differences in screening history, tumour characteristics and survival between women with screen-detected versus not screendetected cervical cancer in the east of The Netherlands, 1992-2001. Eur J Obstet Gynecol Reprod Biol. 2008;139(2):204-9.

45. Priest P, Sadler L, Peters J, Crengle S, Bethwaite P, Medley G, et collab. Pathways to diagnosis of cervical cancer: screening history, delay in follow up, and smear reading. BJOG. 2007;114(4):398-407.

46. Depuydt CE, Arbyn M, Benoy IH, Vandepitte J, Vereecken AJ, Bogers JJ. Quality control for normal liquid-based cytology: rescreening, high-risk HPV targeted reviewing and/or high-risk HPV detection? J Cell Mol Med. 2009;13(9B):4051-60. 